PFC/JA-95-39

\title{
MODE CONVERSION HEATING EXPERIMENTS ON THE TORE SUPRA TOKAMAK
}

\author{
* B. Saoutic, A. Bécoulet, T. Hutter, and D. Fraboulet
}

A. K. Ram and A. Bers

April 1996

\author{
Plasma Fusion Center \\ Massachusetts Institute of Technology \\ Cambridge, Massachusetts 02139 USA
}

* Association Euratom - Commissariat à l'Energie Atomique sur la Fusion, Cadarache, France

This work was supported in part by DOE Grant No. DE-FG02-91ER54109. Reproduction, translation, publication, use and disposal, in whole or part, by or for the United States Government is permitted.

Published in Physical Review Letters 76 (10), 1647 (1996). 


\title{
Mode Conversion Heating Experiments on the Tore Supra Tokamak
}

\author{
B. Saoutic, A. Bécoulet, T. Hutter, and D. Fraboulet \\ Association Euratom-Commissariat à l'Energie Atomique sur la Fusion, C.E. Cadarache, 13 St Paul-lez-Durance, France
}

\author{
A. K. Ram and A. Bers \\ Plasma Fusion Center, Massachusetts Institute of Technology, Cambridge, Massachussets 02139
}

(Received 10 May 1995)

\begin{abstract}
A new scenario for efficient mode conversion heating, in the ion cyclotron range of frequency, is proposed. Experiments on the Tore Supra tokamak demonstrate that this new scheme can be as efficient as minority ion cyclotron heating. A detailed analysis of this mode conversion scenario, using a new complete 1D model and the 3D full-wave code ALCYON, shows a dominant mode conversion fraction regardless of the antenna phasing. It identifies the new scenario to be a "global" resonator, containing mode conversion, coupled to the antenna.
\end{abstract}

PACS numbers: $52.50 . \mathrm{Gj}, 52.40 . \mathrm{Db}, 52.55 . \mathrm{Fa}$

A variety of schemes for radio frequency heating of fusion plasmas in the ion cyclotron range of frequency (ICRF) has been shown to be effective in tokamaks. One of these involves mode conversion (MC) from the fast magnetosonic wave to the slow ion Bernstein wave (IBW) near the ion-ion hybrid resonance of a multispecies plasma. Numerous applications of this scheme are relevant to tokamak reactors: on and off axis electron heating and current drive $[1,2]$, synergism with lower hybrid waves for enhanced current drive $[3,4]$, and channeling of alpha particle power for current drive and increased reactivity [5].

In spite of its important potential applications, $\mathrm{MC}$ has scarcely been studied on the large, present day tokamaks. Previously, using antennas on the high field side (HFS) of the plasma, direct electron heating by IBW's had been observed [6]. In this scenario, $100 \%$ of the fast wave power can be converted to IBW's. However, in present day experiments, the fast wave antennas are placed more conveniently, on the low field side (LFS) of the plasma. In such a configuration, the fast wave encounters a cutoff $n_{\|}^{2}=L$ (LC) and has to tunnel through an evanescent zone before it reaches the ion-ion hybrid layer $S=0$ in the vicinity of which MC occurs (here, we use the notations $n_{\|}^{2}, R, L$, and $S$ as defined by Stix [7]). When only considering this cutoff-resonance pair, the mode converted power can be shown to be given by $T(1-T)$, where $T$ is the power transmission coefficient through the cutoff [8]. Consequently, the maximum power that can be mode converted is only $25 \%$ of the total incident fast wave power. However, recently, some authors (Majeski, Phillips, and Wilson [2] and Ram et al. [4]) have noticed that by including the cutoff $n_{\|}^{2}=R(\mathrm{RC})$ on the HFS of the plasma, one obtains a cutoff-resonance-cutoff triplet forming a resonator (shaded area in Fig. 1) which can be critically coupled to the fast wave, provided a phase condition is fulfilled. It is then possible to show that if $T=0.5$, all the incident fast wave power can be mode converted. Unfortunately, such a search for efficient MC suffers from important drawbacks: the critical coupling is very sensitive to the density profile, the ion species mix, and the launched $n_{\|}$. Consequently, this type of scenario is likely to be restricted to small operating windows in density and to work only for some of the $n_{\|}$launched by the antenna.

Rather than seeking to achieve complete MC with the "internal" resonator system (between the HFS-RC and LC), we propose to rely on the global, external resonator structure formed by the $n_{\|}^{2}=R$ annulus (see Fig. 1) which spans (along the equatorial plane) from the HFS$\mathrm{RC}$ to the LFS-RC near the antenna, and also contains the $\mathrm{MC}$ layer. If the fast wave is coupled to a plasma with no absorption mechanism, it will bounce inside this "global" resonator until the power is dissipated through the weak edge losses (tunneling between the RC and the wall). Now, if any stronger absorption mechanism exists in the plasma, it will damp most of the fast wave energy. It is by using this phenomenon that we get very efficient plasma heating with low single pass absorption schemes like fast wave direct electron heating (FWEH) $[9,10]$.

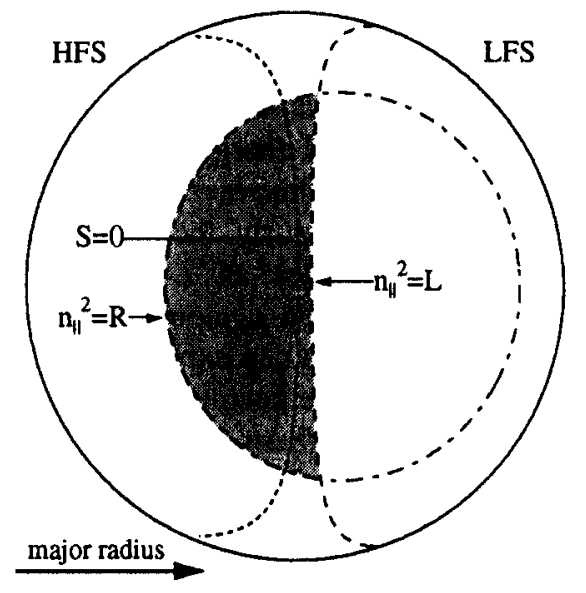

FIG. 1. Poloidal section of the plasma with the cold plasma cutoff and resonant surfaces: the ion-ion hybrid layer (dotted line), the $n_{\|}^{2}=L$ cutoff (dashed line), and the $n_{\|}^{2}=R$ cutoff (dot-dashed line). 
In the same way, by avoiding any competing damping mechanism in the plasma, all the fast wave power will be mode converted to IBW, even if the phase condition for an "internal" resonator is not fulfilled.

We will first describe experiments, using this scheme, on the Tore Supra tokamak where efficient MC heating has been observed. These experimental results are then analyzed using the 3D full-wave code ALCYON [11] and a new complete $1 \mathrm{D}$ model.

Experimental observations. - For the MC experiments, the Tore Supra tokamak was operated with the following parameters: major radius $2.36 \mathrm{~m}$, minor radius $0.72 \mathrm{~m}$, plasma current $0.8 \mathrm{MA}$, toroidal magnetic field at the plasma center $\left(B_{t}\right) 3.85 \mathrm{~T}$, volume averaged density $3.5 \times$ $10^{19} \mathrm{~m}^{-3}$. The plasma composition was a mixture of hydrogen $(\mathrm{H})$ and helium $3\left({ }^{3} \mathrm{He}\right)$ with a small remnant of deuterium (D). The ion species ratio $\rho=n_{\mathrm{H}} /\left(n_{\mathrm{H}}+\right.$ $n^{n^{3} \mathrm{He}}$ ) was chosen to be around 0.5 in order to minimize the possible damping through ion cyclotron resonance. For the same reason, the launched wave frequency was $48 \mathrm{MHz}$, chosen so that the $\mathrm{H}$ and ${ }^{3} \mathrm{He}$ resonance layers are located far from the center, in the colder part of the plasma. The antennas were operated in a dipole configuration $\left(n_{\|} \approx 15\right)$. Measurements of the electron temperature $\left(T_{e}\right)$ and density $\left(n_{e}\right)$ were carried out using a twelve-channel Thomson scattering system, a Michelson interferometer for electron cyclotron emission, and a five chord far infrared interferometer. The ion temperature $\left(T_{i}\right)$ and the isotopic ratio of $\mathrm{H}$ to $\mathrm{D}\left(n_{\mathrm{H}} / n_{\mathrm{D}}\right)$ were measured by a system of five perpendicular and one parallel charge exchange (CX) neutral analyzers. Hot ions were monitored with the $\mathrm{CX}$ analyzers and with the ripple loss diagnostic. The quantity $n_{\mathrm{D}}$ is deduced from the neutron flux and $T_{i}$. Then, $n_{\mathrm{H}}$ is determined from the isotopic ratio. The density of ${ }^{3} \mathrm{He}$ and carbon are inferred from the $Z_{\text {eff }}$ measurement (from visible bremsstrahlung), neglecting impurities other than carbon (this last hypothesis has been checked by spectroscopic measurements).

Figure 2 demonstrates the strong central electron heating which is observed when applying an ICRF pulse at a power level of $2 \mathrm{MW}$. Global confinement studies indicate that the electron stored energy fits the global RebutLallia-Watkins prediction, thus revealing a quite efficient heating scheme. Simultaneously, the thermal energy content (ion+electron) indicates an $L$-mode confinement, equivalent to a minority ion cyclotron heating scheme. Three damping mechanisms can explain the plasma heating: ion cyclotron resonance heating where the suprathermal ions slow down on the thermal bulk, FWEH, and MC. Ion cyclotron resonant damping can be ruled out because, during the heating phase, $T_{e}$ peaks very far from the $\mathrm{H}$ or ${ }^{3} \mathrm{He}$ cyclotron resonance layers. Moreover, very few suprathermal ions are detected by the CX analyzers and by the ripple loss diagnostic. It is possible to discriminate between FWEH and MC by examining their power deposition profiles. The power deposition of FWEH is always

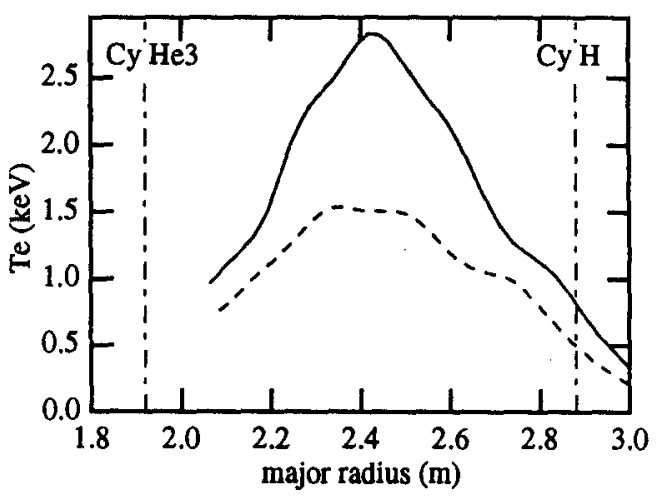

FIG. 2. Electron temperature profile during Ohmic (dashed line) and ICRF (solid line) phases. The dot-dashed lines indicate the $\mathrm{H}$ and ${ }^{3} \mathrm{He}$ fundamental cyclotron resonance layers.

localized at the center of the plasma and is insensitive to the toroidal magnetic field and plasma ion species mix [11]. On the contrary, the power deposition of MC heating depends on the location of the MC layer, which can be controlled by adjusting either $B_{t}$ or $\rho$.

Such experiments, where the location of the MC layer is changed, have been conducted on the Tore Supra tokamak. The ion species mix was varied during a single shot using the following procedure: the plasma is initiated in ${ }^{3} \mathrm{He}$. Then, ${ }^{3} \mathrm{He}$ is puffed at 5.5 and $9.5 \mathrm{~s}$. As ${ }^{3} \mathrm{He}$ is a totally recycling gas, the ${ }^{3} \mathrm{He}$ density increases in successive steps during the shot. In subsequent shots, this ${ }^{3} \mathrm{He}$ puffing is reproduced and the density is controlled by a feedback loop injecting $\mathrm{H}$. Figure 3(a) demonstrates the procedure: the dashed line represents the total number of electrons $\left(N_{e}\right)$

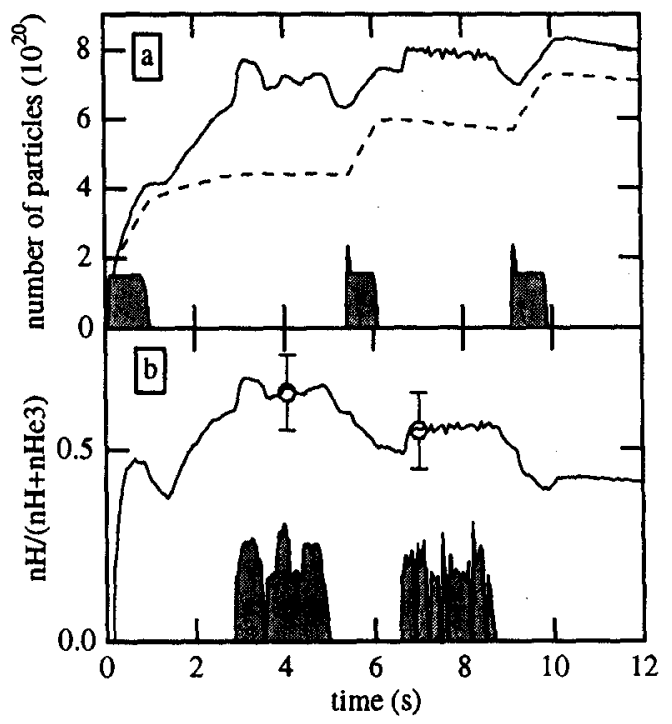

FIG. 3. Evolution of the total number of electrons and ion species mix for shot 15905 . In (a), the solid line indicates $N_{e}$ for shot 15905, the dashed line $N_{e}$ for shot 15901 without $\mathrm{H}$ injection, and the shaded area the ${ }^{3} \mathrm{He}$ puffs. (b) The ion species mix for shot 15905 . The shaded areas indicate the ICRF pulses in a.u. 
during shot 15901 with ${ }^{3} \mathrm{He}$ puffs only, while the solid line represents $N_{e}$ during shot 15905 with ${ }^{3} \mathrm{He}$ puffs and $\mathrm{H}$ feedback. Absolute measurements give $\rho=0.65 \pm 0.1$ during the first ICRF pulse and $0.55 \pm 0.1$ during the second one. Despite the large error bars inherent in the absolute measurements of $\rho$, we can get a more precise evaluation of its relative evolution by assuming that the number of ${ }^{3} \mathrm{He}$ atoms in shot 15905 is given by $N_{e}$ during shot 15901 multiplied by $1-\alpha$ and divided by 2 . The constant $\alpha$ accounts for $\mathrm{H}$ desorption from the wall during shot 15901 and is estimated from the $\rho$ measurement at $t=4 \mathrm{~s}(\alpha=0.17)$. Figure 3(b) displays this estimate, showing that, indeed, $\rho$ decreases between the two ICRF pulses.

Figure 4 shows that, as expected, the shape of the $T_{e}$ profile follows the location of the MC layer, which moves toward the HFS when lowering $B_{t}$ and moves back toward the $\mathrm{H}$ layer on the LFS when decreasing $\rho$. Consequently, the dominant damping scheme is clearly MC. Note on Fig. 4 that the central value of $T_{e}$ is around $2 \mathrm{keV}$ for 1.2 MW injected (shot 15905), for both $\rho=0.55$ and $\rho=0.65$, the power deposition being still central enough not to alter the confinement properties.

Analysis of mode conversion experiments. - The variational full-wave code ALCYON computes the electromagnetic field of the Alfvén compressional magnetosonic mode. The wave can generate torsional and electrostatic modes if a MC process occurs. The effect of MC leads to the development of a sequence of field components with smaller and smaller scales which ultimately are the converted waves at increasing $k_{\perp}$. Such a phenomenon involving two drastically different scale lengths cannot be handled numerically by a finite element code. However, one can take advantage of the fact that the small-scale components are progressively less coupled to the primary magnetosonic wave. This wave can thus safely be computed if the set of decreasing scale components are damped by an ad hoc imaginary functional. This one is built with high-

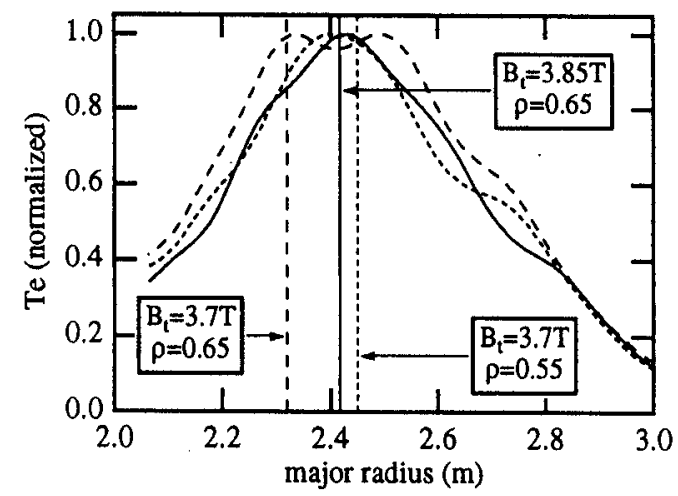

FIG. 4. Normalized electron temperature profile and mode conversion layer location for shot 15910 (solid lines), shot 15905 at $t=4.5 \mathrm{~s}$ (dashed line), and shot 15905 at $t=$ $8.2 \mathrm{~s}$ (dotted line). The mode conversion layer positions are determined using the ALCYON code. order field derivatives; i.e., it artificially absorbs the short wavelength structures. One then gets the magnetosonic field, the coalescence area, and the power going to $\mathrm{MC}$ [12]. Power going to ions (through resonant processes) and to electrons (through direct fast wave heating) is simultaneously determined from the magnetosonic field. This treatment of $\mathrm{MC}$ has been checked by comparing its results to those of a new, simpler 1D model [13]. This 1D model exactly solves the wave equation in the mode conversion region and in the antenna neighborhood and asymptotically connects the two solutions through the WKB approximation. This allows one to take into account the various reflections occurring in the plasma and the interaction of the wave with the antenna.

This procedure has been applied to the analysis of the $\mathrm{MC}$ experiments. Figure 5 displays the power coupled to the plasma for all the damping mechanisms (dipole configuration, shot $15905, t=7 \mathrm{~s}$ ). The fraction of mode converted power is close to $90 \%$ of the launched power regardless of the wave numbers $N_{\varphi}$ that propagate in the plasma. Damping on ion cyclotron resonances is completely negligible. Direct coupling to electrons reaches a maximum fraction of $20 \%$ for the optimum $N_{\varphi}$ where the phase velocity matches the thermal velocity and represents $10 \%$ of the total absorbed power.

The wave numbers launched by the antennas in dipole operation are high $\left(N_{\varphi}\right.$ of the order of $\left.30-40\right)$, so the resultant IBW is excited with a phase velocity lower than the thermal velocity of electrons, and is thus rapidly damped within a few centimeters of the coalescence region. This allows one to identify the coalescence region and power deposition. We then find out that the deposition of mode converted power on electrons is quite central, in good agreement with the peaked $T_{e}$ profile experimentally observed.

Since the internal resonator model of $[2,4]$ requires that, for critical coupling, there be no reflected power at the LC, we should not observe any standing wave structure between the LC and LFS-RC. However, results from our 1D model [13] and from ALCYON (see Fig. 6) show the existence of the global resonator and contradict the

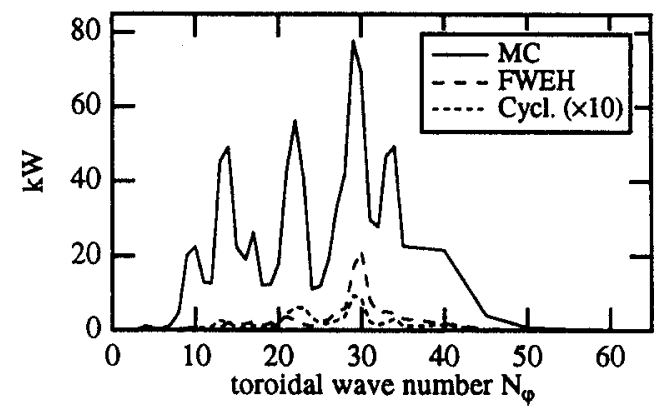

FIG. 5. Power coupled to the plasma for each toroidal number and each damping mechanism: mode conversion (solid line), fast wave direct electron heating (dashed line), and cyclotron resonance (dotted line, $\times 10$ ). 


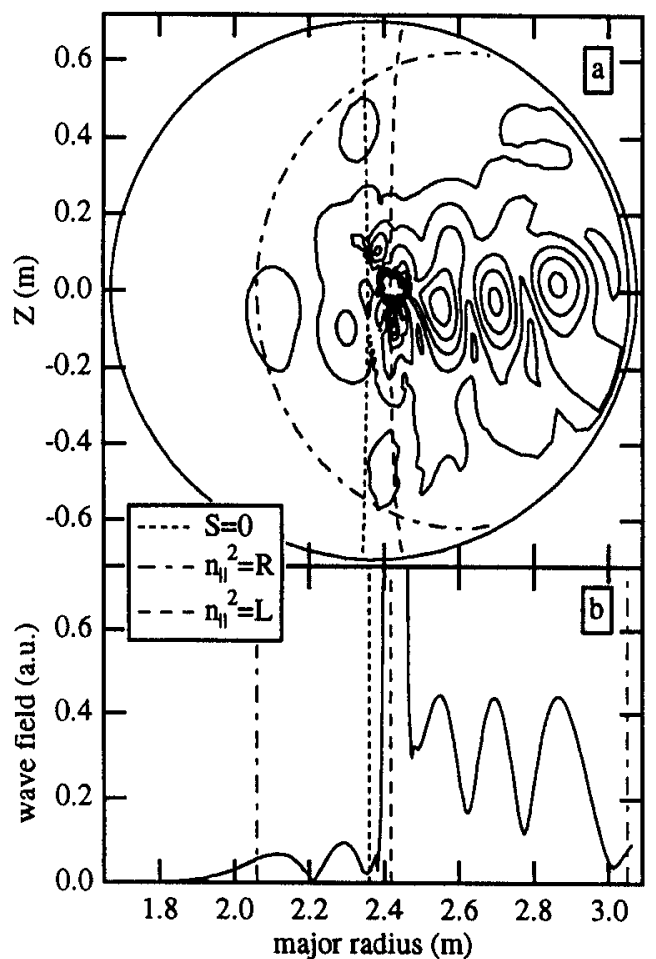

FIG. 6. Poloidal section (a) and equatorial section (b) of the module of the wave field simulated by ALCYON for $N_{\varphi}=30$. Cutoffs and resonances are indicated: the ion-ion hybrid layer (dotted line), the $n_{\|}^{2}=L$ cutoff (dashed line), and the $n_{\|}^{2}=R$ cutoff (dot-dashed line).

scenario of the internal resonator. This is a general feature for all $N_{\varphi}$ propagating in the plasma and manifests itself in the strong resonant peaks in the radiation resistance of the antenna (see Fig. 5). The only damping in the global resonator is due to mode conversion which is in agreement with experiments because the ion resonances are not inside the plasma. The existence of the global resonator also extends the operating regime beyond that for the internal resonator for efficient mode conversion electron heating.

In conclusion, experiments on the Tore Supra tokamak have demonstrated that efficient mode conversion with low field side antennas can be achieved without resorting at any high field side resonator effect. This extends mode conversion scenarios to a much larger operating window in density, the only constraint being to minimize competing damping mechanisms. This scheme does not require a power transmission coefficient of 0.5 lowering the constraint on the ion species mixture. Furthermore, the power fraction which is mode converted is independent of the launched toroidal spectrum, and thus of the antenna phasing. Especially, this scenario allows efficient mode conversion for the low wave numbers that can further accelerate the electrons of the suprathermal tail driven by lower hybrid waves and significantly improve the current drive efficiency [3].

The authors acknowledge the contribution of the ICRF engineering team and the Tore Supra tokamak pilot group whose contributions were essential to this work. We are especially grateful to $\mathrm{C}$. Chamouard and C. Grisolia for their careful management of the ${ }^{3} \mathrm{He}$ injection lines and to J. Harris for fruitful discussion. The work by A. K. Ram and A. Bers was supported in part by a U.S. DOE contract at the Plasma Fusion Center, MIT.

[1] A. K. Ram and A. Bers, Phys. Fluids B 3, 1059 (1991).

[2] R. Majeski, C. K. Phillips, and J.R. Wilson, Phys. Rev. Lett. 73, 2204 (1994).

[3] C. Gormezano, in Radio Frequency in Plasmas, edited by Miklos Porkolab and Joel Hosea, AIP Conf. Proc. No. 289 (AIP, New York, 1993), p. 87.

[4] A.K. Ram et al., in Proceedings of the 21st European Conference on Controlled Fusion and Plasma Physics, Montpellier, France, 1994 (European Physical Society, Geneva, 1994), Vol. 18B, Pt. III, p. 1134.

[5] N.J. Fisch et al., in Proceedings of the 21st European Conference on Controlled Fusion and Plasma Physics, Montpellier, France, 1994 (Ref. [4]), Pt. II, p. 640.

[6] J. Adam, Plasma Phys. Controlled Fusion 29, 443 (1987).

[7] T.H. Stix, Waves in Plasmas (American Institute of Physics, New York, 1992).

[8] V. Fuchs and A. Bers, Phys. Fluids 31, 3702 (1988).

[9] C. C. Petty et al., Phys. Rev. Lett. 69, 289 (1992).

[10] Equipe Tore Supra, Plasma Phys. Controlled Fusion 36 , B123 (1994).

[11] A. Bécoulet et al., Phys. Plasmas 1, 2908 (1994).

[12] A. Bécoulet et al., Association EURATOM-CEA sur la Fusion, C.E. Cadarache, France, DRFC/Note interne No. 1267, 1993.

[13] A. Bers et al. (to be published). 